\title{
SET8 induces epithelial-mesenchymal transition and enhances prostate cancer cell metastasis by cooperating with ZEB1
}

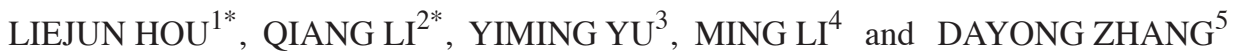 \\ ${ }^{1}$ Department of Urology, The Affiliated Hospital of Medical College, Ningbo University, Ningbo, Zhejiang 315021; \\ ${ }^{2}$ Department of Urology, The First Affiliated Hospital of Shihezi University School of Medicine, Shihezi, Xinjiang 832000; \\ ${ }^{3}$ Department of Respiratory Medicine, The Affiliated Hospital of Medical College, Ningbo University, Ningbo, Zhejiang 315000; \\ ${ }^{4}$ Department of Urology, The Sixth Renmin Hospital of Shanghai Jiaotong University, Shanghai 200233; \\ ${ }^{5}$ Zhejiang University City College, Hangzhou, Zhejiang 310015, P.R. China
}

Received January 18, 2015; Accepted November 6, 2015

DOI: $10.3892 / \mathrm{mmr} .2015 .4733$

\begin{abstract}
Mounting evidence suggested that histone H4K20-specific methyltransferase SET8 is required to maintain the malignant phenotype of various cancer types; however, the role of SET8 in mediating tumor metastasis in prostate cancer ( $\mathrm{PCa}$ ) has remained elusive. The present study demonstrated that small interfering RNA-mediated knockdown of SET8 inhibited the invasive potential of the PCa cell line PC-3 in vitro. Knockdown of SET8 reduced sphere formation, downregulated E-cadherin and $\alpha$-catenin, and upregulated $\mathrm{N}$-cadherin and vimentin expression in $\mathrm{CaP}$ cells, while upregulation of SET8 expression with a recombinant plasmid had the opposite effect. Furthermore, SET8 was shown to be physically associated with the epithelial-mesenchymal transition (EMT) inducer zinc finger E-box-binding homeobox 1 (ZEB1) in PCa cell lines. Chromatin immunoprecipitation suggested that SET8 binds to the promoter of cell adhesion molecule E-cadherin and vimentin. Luciferase reporter assays suggested that E-cadherin and vimentin are direct targets of SET8; furthermore, loss- and gain-of function studies of SET8 and ZEB1 indicated that suppression of downstream E-cadherin and activation of vimentin are important mechanisms by which SET8 and ZEB1 cooperatively trigger metastasis. Furthermore, SET8-induced methylated H4K20 was indicated to exert a dual function in ZEB1-regulated gene expression. In conclusion, the present study revealed that SET8 and ZEB1 are functionally interdependent in promoting
\end{abstract}

Correspondence to: Professor Liejun Hou, Department of Urology, The Affiliated Hospital of Medical College, Ningbo University, 247 Renmin Road, Jiangbei, Ningbo, Zhejiang 315021, P.R. China E-mail: liejunhou001@163.com

*Contributed equally

Key words: SET8, zinc finger E-box-binding homeobox 1, metastasis, E-cadherin, vimentin the EMT and enhancing the invasive potential of PCa cells in vitro.

\section{Introduction}

Prostate cancer ( $\mathrm{PCa}$ ) is the second leading cause of cancer-associated mortality in men in the US, and up to $40 \%$ of PCa cases eventually develop metastasis (1). Metastasis remains a significant clinical challenge in various cancer types, as it is the major cause of mortality and is responsible for low five-year survival rates (2). Epithelial-mesenchymal transition (EMT) is thought to be the initial step of tumor metastasization, as it renders cancer cells with migratory and invasive properties required to detach from the primary tumor and enter vessels (3). Downregulation of E-cadherin and upregulation of $\mathrm{N}$-cadherin are considered as hallmarks of EMT $(4,5)$.

Several transcription factors that induce EMT, such as SNAI1 and SNAI2/SLUG, two members of the Snail superfamily of zinc finger transcriptional repressors $(6,7)$, have been shown to act as E-cadherin repressors. Furthermore, zinc finger E-box-binding homeobox 1 (ZEB1) and ZEB2, which belong to the ZEB family, have emerged as key factors that regulate E-cadherin and the induction of the EMT $(8,9)$. In addition, certain basic helix-loop-helix factors, including E47 and TWIST1, function as EMT regulators affecting tumor cell invasion and metastasis (10). Vimentin has been demonstrated to significantly contribute to the invasive phenotype of $\mathrm{PCa}$ cells $(11,12)$. EMT of PCa cells involves the acquisition of mesenchymal markers such as vimentin and differentiation into a phenotype with an increased probability of metastasis formation.

Histone modifications, including methylation, acetylation, phosphorylation and ubiquitination, are considered to be epigenetic variations linked with processes of carcinogenesis and cancer progression, including EMT, and modification of gene expression; furthermore, they appear to have a prognostic value $(13,14)$. In these modifications, histone methylation has been proved to have important roles in various aspects of chromatin function. Methylation of $\mathrm{H} 3 \mathrm{~K} 9$ and $\mathrm{H} 3 \mathrm{~K} 27$ is associated with gene silencing, whereas methylation of H3K4, H3K36 and 
H3K79 has been linked to transcriptional activation $(15,16)$. However, the effects of methylation of H4K20 to form monomethylated H4K20 (H4K20me1) on gene transcription requires further study. SET8, also known as PR-SET7/KMT5A, is a histone H4K20-specific methyltransferase (17), which specifically monomethylates H4K20 (18). SET8 was reported to exert a dual role on transcription by the activation of $\mathrm{N}$-cadherin and repression of E-cadherin (19). Of note, SET8 has been reported to be involved in activation as well as repression of transcription $(20,21)$. SET8, through its methylase activity, has been implicated in a variety of biological processes, including transcriptional regulation, genomic stability, heterochromatin formation and cell-cycle progression, while the role of SET8 in $\mathrm{PCa}$ and the underlying mechanisms have remained poorly understood. The present study aimed to shed light on the role of SET8 in the cancer progression and EMT of PCa cells; furthermore, the implication of ZEB1 and H4K20me1 was evaluated.

\section{Materials and methods}

Reagents. Anti-human SET8 antibody (cat. no. sc-135009, rabbit $\operatorname{IgG}, 1: 500$ ), ZEB1 antibody (cat. no. sc-25388, rabbit IgG, 1:1,000), E-cadherin antibody (cat. no. sc-21791, mouse IgG1, 1:1,000), N-cadherin antibody (cat. no. sc-7939, rabbit IgG, 1:1,000), $\alpha$-catenin antibody (cat. no. sc-107193, goat IgG, 1:1,000), vimentin antibody (cat. no. sc-373717, mouse IgG1, 1:500), $\beta$-actin antibody (cat. no. sc-7210, rabbit IgG, 1:500) and secondary antibodies (cat. no. sc-2039, goat anti-mouse IgG-B, 1:2,000; and cat. no. sc-2040, goat anti-rabbit IgG-B, 1:2,000) were all purchased from Santa Cruz Biotechnology, Inc. (Dallas, TX, USA). Anti-H4K20me1, anti-H4K20me2, anti-H4K20me3 and anti-H4 were obtained from Abcam (Cambridge, MA, USA). Specific small interfering (si)RNAs targeting SET8 or ZEB1 (SiSET8\#1: 5'-CCGGTTGAACAG ATGGCCTTATATTCTCGAGAATATAAGGCCATC TGTTCAATTTTTG-3'; SiSET8\#2: 5'-CCGGGCCTAGGA AGACTGATCAATCCTCGAGGATTGATCAGTCTTCCT AGGCTTTTTG-3'; SiZEB1\#1: 5'-CCGGGTCTGGGTGTA ATCGTAAATTCTCGAGAATTTACGATTACACCCAGA CTTTTTG-3'; and SiZEB1\#2: 5'-CCGGCTGAACCTCAG ACCTAGTAATCTCGAGATTACTAGGTCTGAGGTTCA GTTTTTG-3') were purchased from Sigma-Aldrich (St. Louis, MO, USA). A negative control siRNA used as control was also from Sigma-Aldrich. Matrigel ${ }^{\circledR}$ was purchased from BD Biosciences (Franklin Lakes, NJ, USA). The restriction enzymes were from New England Biolabs, Inc. (Ipswich, MA, USA). Lipofectamine $2000^{\mathrm{TM}}$ and Lipofectamine RNAimax (Invitrogen; Thermo Fisher Scientific, Inc., Waltham, MA, USA) were used for the transfection experiments. A Renilla-Glo ${ }^{\mathrm{TM}}$ Luciferase assay system kit (Promega Corp. Madison, WI, USA) was used for the luciferase assay.

Cell culture. The PC-3 androgen-non-responsive PCa cell line and the PZ-HPV-7 non-transformed prostate epithelial cell line were obtained from the American Type Culture Collection (Manassas, VA, USA). The cells were cultured in Dulbecco's modified Eagle's medium (DMEM; HyClone; GE Healthcare, Little Chalfont, UK) supplemented with $10 \%$ fetal bovine serum (FBS; HyClone). The LNCaP androgen-responsive
PCa cell line (also obtained from the American Type Culture Collection) was cultured in RPMI-1640 with 10\% FBS, 100 units of penicillin/streptomycin (Invitrogen; Thermo Fisher Scientific, Inc.) and maintained at $37^{\circ} \mathrm{C}$ in a humidified atmosphere containing $5 \% \mathrm{CO}_{2}$.

Reverse-transcription quantitative polymerase chain reaction (RT-qPCR). Total RNA of cell lysates was extracted with TRIzol solution (Invitrogen) at $48 \mathrm{~h}$ post-transfection and reverse-transcribed into cDNA using $1 \mu \mathrm{g}$ total RNA with Moloney murine leukemia virus reverse transcriptase (Beijing TransGen Biotech Co., Ltd., Beijing, China), according to the manufacturer's protocol. Real-time PCR primers (Invitrogen; Thermo Fisher Scientific, Inc.) were as follows: SET8 forward, 5'-TATCACTCTGTTTCACGCCA-3' and reverse, 5'-ACC ATTCCTCCATCTCATCC-3'; $\beta$-actin forward, 5'-TGGCAC CCAGCACAATGAA-3' and reverse, 5'-CTAAGTCATAGT CCGCCTAGAAGCA-3'. Real-time PCR was performed using SYBR Green (Roche Diagnostics, Basel, Switzerland) in an ABI 7500 sequence detection system (Applied Biosystems; Thermo Fisher Scientific). The PCR reaction conditions were as follows: An initial stage of $95^{\circ} \mathrm{C}$ for 2 mins; then 35 cycles of $95^{\circ} \mathrm{C}, 1 \mathrm{~min}, 55^{\circ} \mathrm{C}, 1 \mathrm{~min}, 72^{\circ} \mathrm{C}, 1 \mathrm{~min}$; and finally, $72^{\circ} \mathrm{C}$ for $10 \mathrm{~min}$, with a $4^{\circ} \mathrm{C}$ pause. The $2^{-\Delta \Delta \mathrm{Cq}}$ method was used. All experiments were performed in triplicate with $\beta$-actin used as a normalization control.

Western blot analysis. Cells were lysed in $50 \mathrm{ml}$ lysis buffer [100 mM Tris- $\mathrm{HCl}$ (pH 7.4), 10 mM ethylenediaminetetraacetic acid (EDTA), 4\% sodium dodecyl sulfate (SDS) and 10\% glycine; Sigma-Aldrich] on ice for $45 \mathrm{~min}$. Following centrifugation at $12,000 \mathrm{x} \mathrm{g}$ for $30 \mathrm{~min}$ at $4^{\circ} \mathrm{C}$, the supernatants were collected and the protein concentration was determined using the bicinchoninic acid (BCA) method, with a commercially available BCA kit (GE Healthcare Life Sciences, Logan, UT, USA). Equal amounts (40 $\mu \mathrm{g}$ protein) of lysate were separated by $8-10 \%$ SDS-polyacrylamide electrophoresis (PAGE) on an SDS-PAGE gel (Invitrogen; Thermo Fisher Scientific, Inc.). The proteins were electro-transferred onto a nitrocellulose membrane (GE Healthcare Life Sciences) using an electro-blotting apparatus (Biorad Mini-Protean Tetra Electrophoresis system; Bio-Rad Laboratories, Inc., Hercules, CA, USA). For Western blot analysis, membranes were blocked with milk and then incubated with the appropriate antibodies for $1 \mathrm{~h}$ at room temperature, followed by five washes with washing buffer (Tris-buffered saline/Tween 20 buffer; Sigma-Aldrich), prior to incubation with a secondary antibody. Immunoreactive bands were visualized using Western blotting Luminal reagent (Santa Cruz Biotechnology, Inc.) according to the manufacturer's protocol, using Kodak X-OMAT BT film (Kodak, Rochester, NY, USA). $\beta$-actin was used as a loading control.

Immunoprecipitation (IP). Cells were washed with cold phosphate-buffered saline and lysed with cold lysis buffer (Sigma-Aldrich; as detailed above) at $4^{\circ} \mathrm{C}$ for $45 \mathrm{~min}$. Whole-cell lysates were incubated with appropriate primary antibodies (the SET8 or the ZEB1 antibody) or normal rabbit/mouse immunoglobulin G (IgG; Santa Cruz Biotechnology, Inc.) with agitation overnight at $4^{\circ} \mathrm{C}$, followed by addition of protein $\mathrm{A} / \mathrm{G}$ Sepharose CL-4B beads (GE Healthcare Life Sciences) for $2 \mathrm{~h}$ 
at $4^{\circ} \mathrm{C}$. Beads were then washed five times with lysis buffer (50 mM Tris-Cl, pH 7.4, $150 \mathrm{mM} \mathrm{NaCl,} 1 \mathrm{mM}$ EDTA, 1\% Nonidet P-40, $0.25 \%$ sodium deoxycholate and protease inhibitor mixture; Sigma-Aldrich)). The immune complexes were subjected to $10 \%$ SDS-PAGE, followed by immunoblotting with secondary antibodies.

Construction of E-cadherin or vimentin reporter plasmid and luciferase reporter assay. For vimentin reporter plasmid construction, a sequence of the vimentin promoter and partial first exon (-736 to +164 bp) was obtained by PCR using specific primers; the E-cadherin reporter was generated as described previously (19). These PCR products were ligated into the pGL3 basic vector to generate pGL3-vimentin or pGL3-E-cadherin luciferase reporter constructs (Promega Corp., Madison, WI, USA) according to the manufacturer's protocol. Cells in 96-well plates were transfected with vimentin or E-cadherin promoter luciferase reporter, Renilla plasmid and the indicated expression constructs using Lipofectamine LTX-Plus (Invitrogen). The amount of DNA was $2 \mu \mathrm{g}$ in each transfection. Forty-eight hours after transfection, the firefly and Renilla luciferases were assayed according to the manufacturer's protocol (Promega) with Renilla luciferase plasmid as a transfection efficiency normalization control. Each experiment was repeated in triplicate.

Chromatin IP (ChIP). ChIP assays were performed according to the manufacturer's protocol (Upstate Biotechnology, Inc., Lake Placid, NY, USA). The chromatin was obtained according to the manufacturer's protocol (Upstate Biotechnology, Inc.). Chromatin was incubated with $3 \mu \mathrm{g}$ SET8 antibody, ZEB1 antibody, H4K20me1 antibody, H4K20me2 antibody, H4K20me3 antibody, anti-H4 or normal rabbit IgG as a negative control, at $4^{\circ} \mathrm{C}$ overnight. Immunoprecipitated DNA was purified using the Qiagen PCR purification kit (Qiagen, Hilden, Germany). qChIP was performed by qPCR using specific primers as stated below. For common ChIP assays, the final target DNA sequence was amplified and resolved on standard agarose DNA gels (Invitrogen; Thermo Fisher Scientific, Inc.). For the DNA amplification, the PCR reaction conditions were: An initial stage of $95^{\circ} \mathrm{C}$ for 2 mins; then 35 cycles of $95^{\circ} \mathrm{C}, 1 \mathrm{~min}$, $55^{\circ} \mathrm{C}, 30 \mathrm{sec}, 72^{\circ} \mathrm{C}, 1 \mathrm{~min}$; and finally, $72^{\circ} \mathrm{C}$ for $10 \mathrm{~min}$, with a $4^{\circ} \mathrm{C}$ pause.

Primers (Invitrogen; Thermo Fisher Scientific, Inc.) used for common ChIP were as follows: E-cadherin forward, 5'-GAGACTGGCACAGTAATCTTC-3' and reverse, 5'-TGG CTAACGCAGTGAAAC-3'; $\alpha$-catenin forward, 5'-TGGTCC TATTGCCCTTTG-3' and reverse, 5'-GCCCTTACCGTGTTT ACC-3'; N-cadherin forward, 5'-GCACTCAGAACAGGC ACAT-3' and reverse, 5'-GCCCAGGAGTTCGAGACCA-3'; vimentin forward, 5'-CCCTGCCTTAGTCTCCCA-3' and reverse, 5'-CTCCTCCTTCCAACCTGTC-3'.

qChIP primers: E-cadherin forward, 5'-AACCCAGTG GAATCAGAAC-3' and reverse, 5'-ATAGACGCGGTG ACCCTC-3'; $\alpha$-catenin forward, 5'-AGAGGGTAAACA CGGTAAGG-3' and reverse, 5'-TCACGGGATGATGAA TAAGA-3'; N-cadherin forward, 5'-AATCCTCCCACTTCA GCC-3' and reverse, 5'-AGCCCAGGAGTTCGAGAC-3'; vimentin forward, 5'-AGCCTATCACAGCCCAGAG-3' and reverse, 5'-CCCATAGCCGATTCCTCA-3'.
Transwell invasion assay. The invasive ability of the cells was investigated using Transwell inserts for 24-well plates $(8 \mu \mathrm{m}$ pore size; EMD-Millipore, Billerica, MA, USA). First, the surfaces of the membranes were coated with $100 \mu$ l Matrigel ${ }^{\circledR}$ $(50 \mu \mathrm{g} / \mathrm{ml})$ for $1 \mathrm{~h}$. A total of $5 \times 10^{4}$ cells in $500 \mu \mathrm{l}$ serum-free medium was added to each upper chamber, while $500 \mu \mathrm{l}$ DMEM containing $10 \%$ FBS was added to the lower compartment. Following incubation for $24 \mathrm{~h}$, the cells on the upper surface of the membrane were removed using cotton swabs, while the cells that had transgressed through the Matrigel-coated membrane to the lower surface were fixed with $2 \%$ paraformaldehyde and stained with crystal violet (Sigma-Aldrich). Images were captured using an inverted microscope (Olympus BX46; Olympus Corp., Tokyo, Japan) at x100 magnification. Quantification of invaded cells in each well was performed in three randomly selected fields. Each experiment was performed in triplicate and representative images are shown.

Statistical analysis. Values are expressed as the mean \pm standard deviation of three independent experiments, using the paired t-test to compare the mean values ( \pm standard deviation). SPSS version 17.0 software was used for the statistical analysis (SPSS, Inc., Chicago, IL, USA). P $<0.05$ was considered to indicate a statistically significant difference.

\section{Results}

Knockdown of SET8 in PC-3 cells. As shown in Fig. 1A, PC-3 cells were transfected with control siRNA, siSET8\#1 or siSET8\#2. Knockdown of SET8 was confirmed at the mRNA and protein level. RT-qPCR showed that the mRNA levels of SET8 were significantly reduced following transfection with siSET8\#1 or siSET8\#2 $(\mathrm{P}<0.05)$ (Fig. 1A, left panel). Concomitantly, western blot analysis showed that in siSET8-transfected PC-3 cells, SET8 was markedly reduced as compared with that in the control siRNA-transfected cells (Fig. 1A, middle panel). While the results confirmed that the two SET8-specific siRNA-containing vectors were successfully constructed and transfected into PC-3 cells, the knockdown efficiency of siSET8\#2 was higher than that of siSET8\#1. Furthermore, western blot analysis confirmed that SET8 was markedly overexpressed in PC-3 cells transfected with specific overexpression vector (Fig. 1A, right panel).

SET8 promotes PCa-cell metastasis and EMT. To assess the effects of SET8 on cell invasion, a Transwell assay was performed. The number of migrated cells transfected with siSET8\#2 accounted for one third of those transfected with control siRNA, while SET8 overexpression increased cell invasion to 3.5 times of that of the control cells (Fig. 1B). These results indicated that SET8 is involved in metastasis of PCa. Considering EMT as the initiation step of metastasis, the present study further examined the role of SET8 in this process. In SET8-overexpressing PC-3 cells, the expression of the epithelial markers $E$-cadherin and $\alpha$-catenin was downregulated, while the mesenchymal markers $\mathrm{N}$-cadherin and vimentin were elevated as indicated by RT-qPCR (Fig. 1C, left panel) and western blot analysis (Fig. 1C, right panel). Conversely, following SET8 knockdown in PC-3 cells, E-cadherin and $\alpha$-catenin were upregulated, while $\mathrm{N}$-cadherin and vimentin 
A
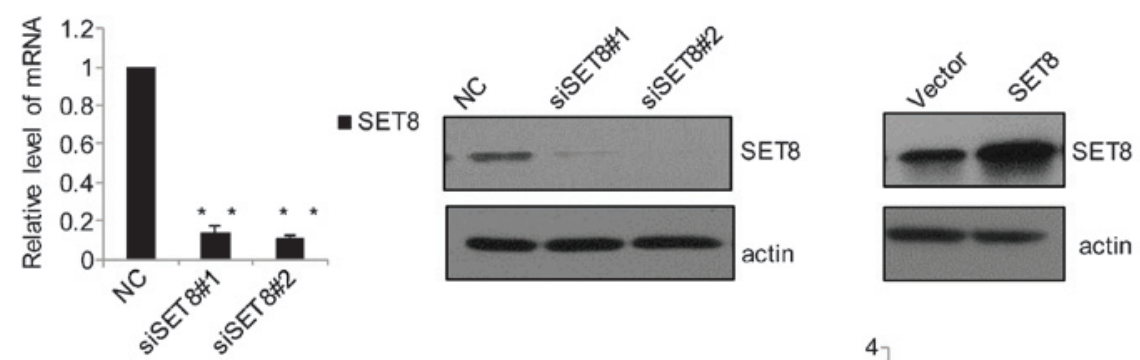

$\mathbf{B}$
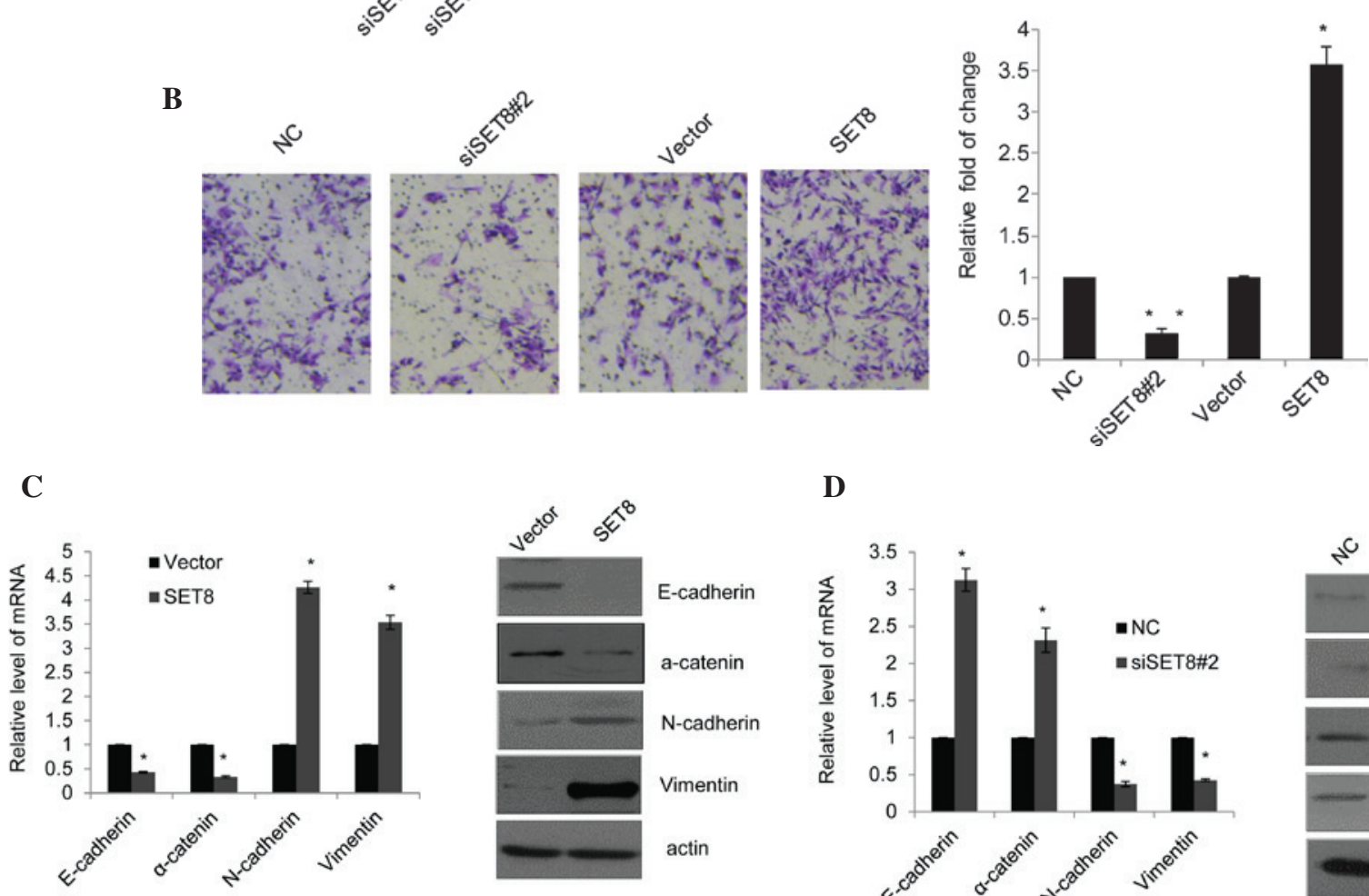

D

$\mathbf{E}$
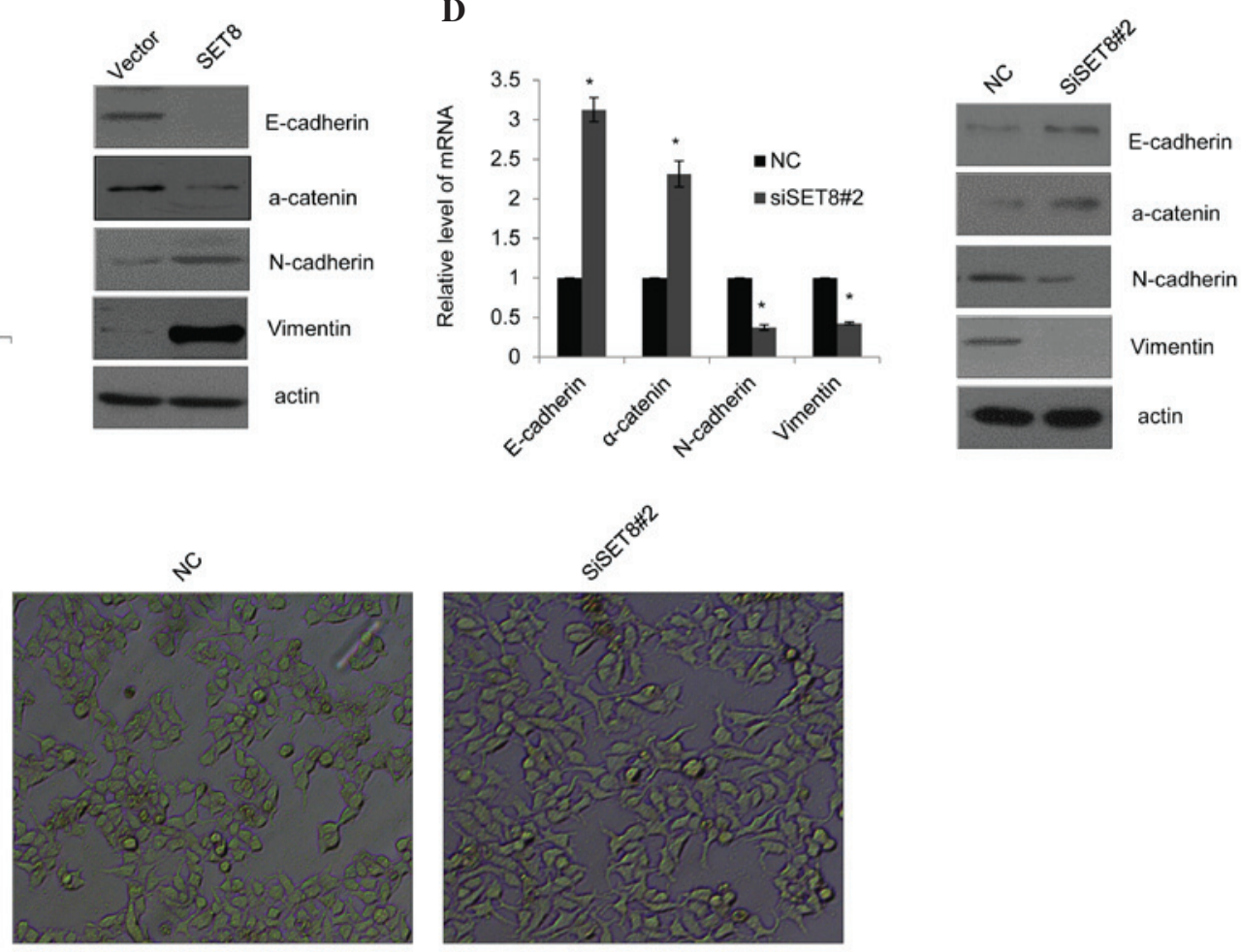

Figure 1. SET8 promotes prostate cancer cell metastasis and epithelial-mesenchymal transition. (A) The SET8 knockdown efficiency was confirmed by qPCR (left panel) and western blot analysis (middle panel), and the SET8 overexpression efficiency was determined by western blot analysis (right panel) in PC-3 cells. (B) PC-3 cells were transfected with control siRNA, siSET8\#2, empty vector or SET8 overexpression vector for 48 h. Cells were then starved for $18 \mathrm{~h}$ prior to cell invasion assays. The invaded cells were stained with crystal violet and counted under a microscope (magnification, x100). Representative images for each group are shown and quantitative results are presented as the fold change compared with the vector group. (C) Effects of SET8 overexpression in PC-3 cells on the mRNA and protein expression of the epithelial markers E-cadherin and a-catenin as well as mesenchymal markers N-cadherin and vimentin as examined by RT-qPCR (left) and western blot analysis (right), respectively. (D) Effects of SET8 knockdown in PC-3 cells on the expression of the indicated epithelial or mesenchymal markers as examined by RT-qPCR (left) and western blot analysis (right), respectively. Values are expressed as the mean \pm standard deviation for triplicate measurements. ${ }^{*} \mathrm{P}<0.05 ;{ }^{* *} \mathrm{P}<0.01$ vs. vector/NC group. (E) PZ-HPV-7 cells (magnification, $\mathrm{x} 10$ ) were treated with specific siRNA against SET8, and the morphological alterations of these cells were examined by phase-contrast microscopy. siRNA, small interfering RNA; $\mathrm{NC}$, negative control; RT-qPCR, reverse-transcription quantitative polymerase chain reaction.

were decreased (Fig. 1D). In addition, observation of PZ-HPV-7 prostate epithelial cells by phase-contrast microscopy showed that depletion of SET8 led to obvious morphological alterations (Fig. 1E).
SET8 is physically associated with ZEB1 but not ZEB2 in PCa cell lines. In order to investigate the mechanism of the role of SET8 in EMT and metastasis, a co-IP assay was performed to assess the interaction between SET8 and various transcription 

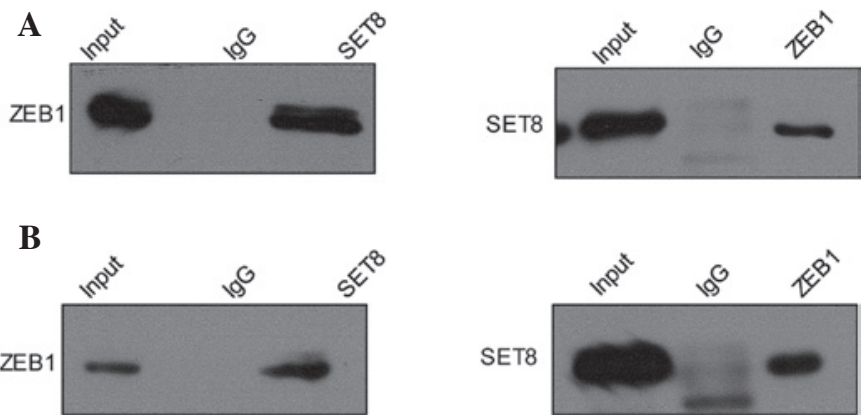

Figure 2. SET8 is physically associated with ZEB1 but not ZEB2 in prostatic cancer cell lines. (A) Co-immunoprecipitation analysis of the association between SET8 and ZEB1. Whole-cell lysates of PC-3 cells were immunoprecipitated with antibodies against the indicated proteins, with $\operatorname{IgG}$ as the negative control and whole lysates as the positive control. The immunocomplexes were then immunoblotted using the indicated antibodies. (B) Whole-cell lysates of LNCaP cells were further used to detect the association between SET8 and ZEB1. IgG, immunoglobulin G; ZEB1, zinc finger E-box-binding homeobox 1.

factors, which have been confirmed to have pivotal roles in tumor metastasis by promoting EMT. Total protein extracts from PC-3 cells were prepared and co-IP experiments were performed with specific antibodies against target proteins. First, IP with anti-SET8 followed by immunoblotting (IB) with the anti-ZEB1 in PC-3 cells indicated that SET8 co-immunoprecipitated with ZEB1 (Fig. 2A, upper panel), and similar results were obtained for IP with anti-ZEB1 followed by IB with anti-SET8 (Fig. 2A, lower panel). This in vitro interaction of SET8 and ZEB1 was also demonstrated in the LNCaP cell line (Fig. 2B). Co-IP experiments using antibodies against other transcription factors, including SNAI1 and ZEB2, showed no interaction with SET8 (data not shown).

SET8 directly regulates $E$-cadherin and vimentin transcription. To further assess the molecular mechanism of the regulatory roles of SET8 in transcription, qChIP assays were performed in PC-3 cells. Several key genes of molecular pathways involved in the EMT and metastasis, namely E-cadherin, $\alpha$-catenin, $\mathrm{N}$-cadherin vimentin and actin, were selected for detection of possible binding to SET8 with their gene promoter regions. The binding of SET8 to the promoter sequences of E-cadherin and vimentin was obviously higher than that to normal IgG (Fig. 3A). Similar results were obtained in ChIP PCR assays in PC-3 cells, as bright bands were obtained in the anti-SET8 groups when primers for the E-cadherin or vimentin promoter were used, while no bands were obtained with normal IgG (Fig. 3B).

To assess whether SET8 directly targeted the promoter regions of E-cadherin and vimentin, luciferase reporter assays were performed in PC-3 cells. E-cadherin or vimentin promoter-driven luciferase reporter vectors were transfected into PC-3 cells with simultaneous SET8 overexpression or depletion. The results indicated that SET8 overexpression or knockdown resulted in repressed or enhanced E-cadherin reporter activity, respectively (Fig. 3C). By contrast, overexpression or silencing of SET8 led to a significant activation or repression, respectively, of the vimentin reporter vector (Fig. 3D).
A

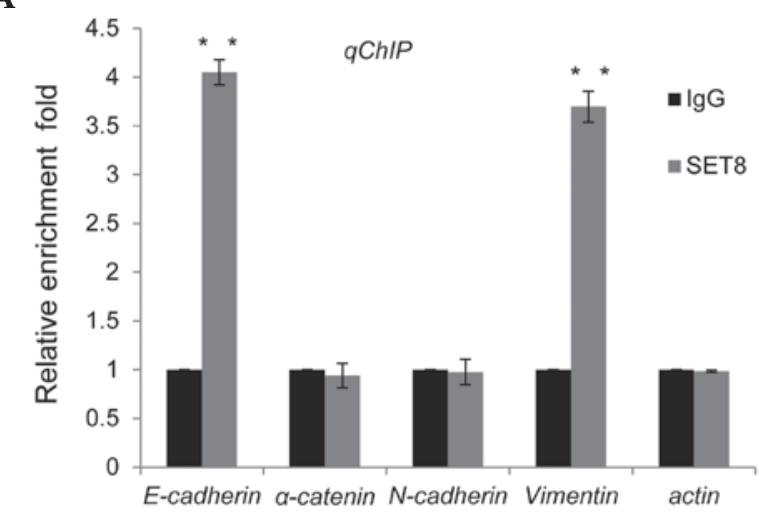

B

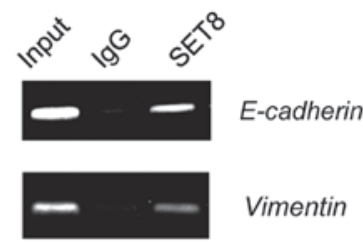

C

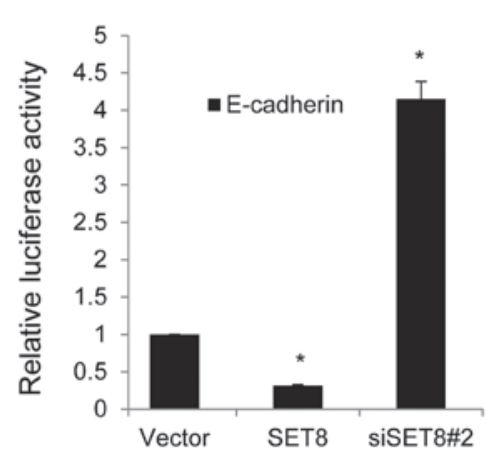

D

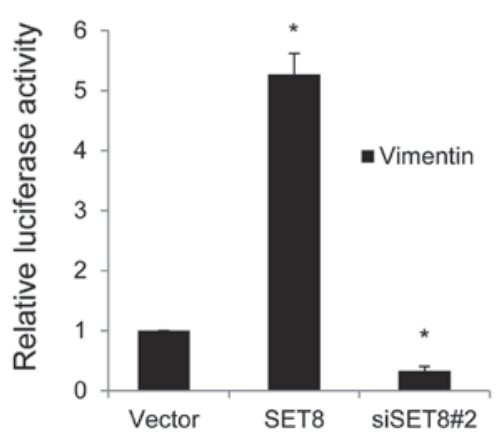

Figure 3. SET8 regulates E-cadherin and vimentin transcription. (A) Recruitment of SET8 to E-cadherin and vimentin promoters. qChIP experiments were performed on PC-3 cells with the indicated antibodies. (B) ChIP assays were performed on PC-3 cells with indicated antibodies to further confirm the results of A. (C) SET8 directly targets the E-cadherin promoter. PC-3 cells were transfected with luciferase reporter constructs containing an E-cadherin promoter sequence along with SET8 overexpression or silencing. Luciferase activities were measured and normalized to those of Renilla. (D) SET8 directly targets the vimentin promoter. A luciferase reporter plasmid containing a vimentin promoter sequence along with SET8 overexpression or silencing vectors were transfected into PC- 3 cells and luciferase activity was assessed. Values are expressed as the mean \pm standard deviation of three independent experiments. ${ }^{*} \mathrm{P}<0.05 ;{ }^{* *} \mathrm{P}<0.01$ vs. vector group. IgG, immunoglobulin G; qChIP, quantitative chromatin immunoprecipitation; siSET8, small interfering RNA specific for SET8. 


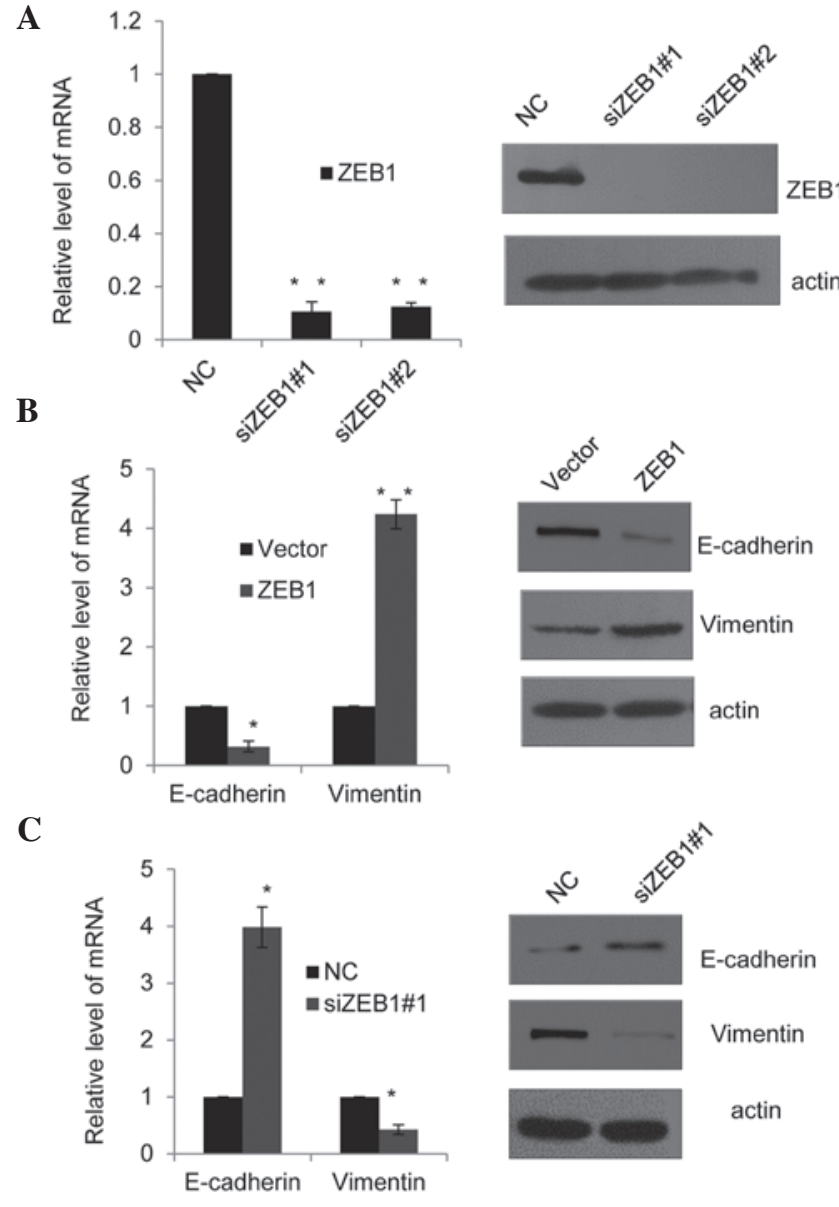

Figure 4. SET8 cooperates with ZEB1 to affect the expression of E-cadherin and vimentin. (A) The knockdown efficiencies of siZEB1\#1 and siZEB1\#2 were confirmed in PC-3 cells by quantitative reverse-transcription polymerase chain reaction with GAPDH used as a normalization control (left panel) and by western blot analysis with $\beta$-actin as a control (right panel). (B) The mRNA and protein levels of E-cadherin and vimentin were detected 24-48 h after transfection of PC-3 cells with ZEB1 constructs. (C) The mRNA and protein levels of E-cadherin and vimentin were detected $48-72 \mathrm{~h}$ after transfection of PC-3 cells with siZEB1. Values are expressed as the mean \pm standard deviation of three experiments. ${ }^{*} \mathrm{P}<0.05 ;{ }^{* *} \mathrm{P}<0.01$ vs. vector/NC. NC, negative control; siZEB1, small interfering RNA specific for ZEB1; ZEB1, zinc finger E-box-binding homeobox 1.

SET8 cooperates with ZEB1 to affect the expression of $E$-cadherin and vimentin. Since the recruitment of ZEB1 to the promoter of E-cadherin had been previously reported (22), the present study assessed whether SET8 and ZEB1 have similar or joint effects on E-cadherin expression patterns, and whether they jointly affect vimentin expression. First, the efficiency of two different ZEB1-specific siRNAs was assessed using RT-qPCR and western blot analysis in PC-3 cells (Fig. 4A). ZEB1 siRNA\#2 was revealed to slightly more efficiently silence ZEB1 than ZEB1 siRNA\#1 and was therefore selected for use in the subsequent experiment. Consistent with SET8 promoter occupancy (Fig. 3A and B) and the effects of SET8 on the expression of the indicated epithelial or mesenchymal markers (Fig. 1C and D). In ZEB1-overexpressing PC-3 cells, the mRNA (Fig. 4B, left panel) and protein expression (Fig. 4B, right panel) of E-cadherin was decreased, while in ZEB1-silenced PC-3 cells, E-cadherin was increased at the mRNA and protein level (Fig. 4C). Furthermore, ZEB1 overexpression enhanced vimentin mRNA and protein
$\mathbf{A}$

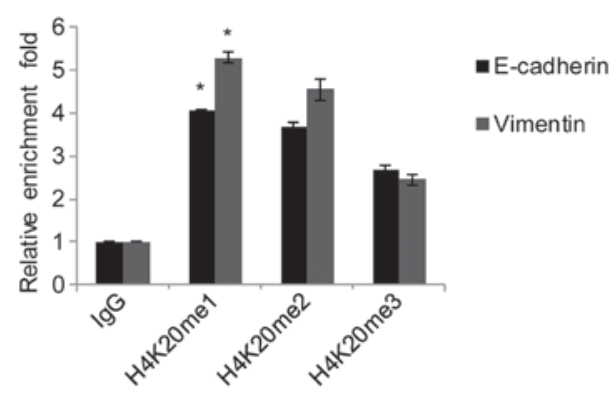

B

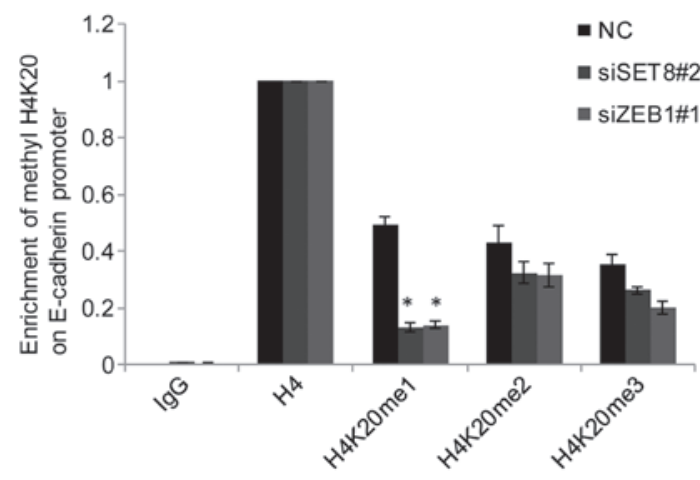

C

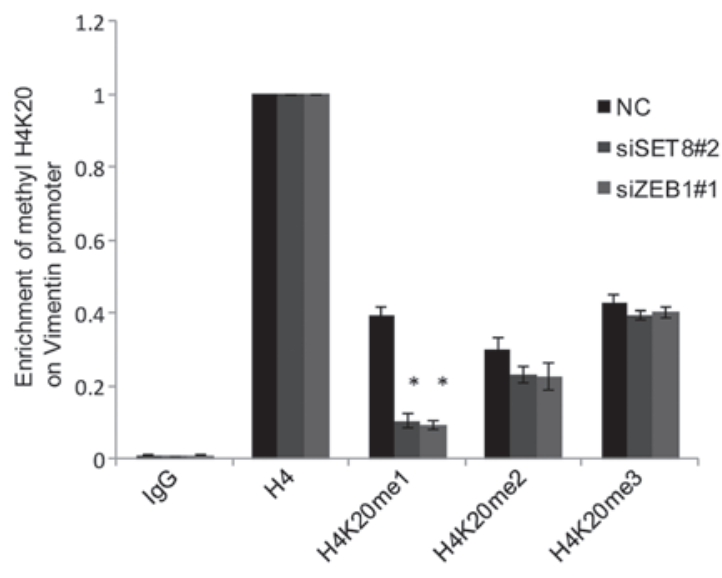

Figure 5. SET8-mediated H4K20me1 has a dual function in ZEB1-regulated gene expression. (A) qChIP assays of the promoter of E-cadherin and vimentin were performed with anti-H4K20me1, anti-H4K20me2 and anti-H4K20me3 with normal IgG used for the negative control. (B) SET8 was silenced using siRNA in PC-3 cells, and qChIP of the promoter of E-cadherin and vimentin was performed with antibodies against H4K20me1, H4K20me2 and H4K20me3 with normalization to H4 as the control. (C) In ZEB1-depleted PC-3 cells, qChIP assays of the promoter of E-cadherin and vimentin were performed with mono-, di-, and tri-methylated H4K20-specific antibodies, and anti-H4 set as $100 \%$. Values are expressed as the mean \pm standard deviation of three experiments. "P<0.05 vs. NC or IgG. IgG, immunoglobulin G; qChIP, quantitative chromatin immunoprecipitation; siSET8, small interfering RNA specific for SET8; NC, negative control; H4K20me1/2/3, mono/di/trimethylated histone H4K20; ZEB1, zinc finger E-box-binding homeobox 1.

expression, while in ZEB1-depleted PC-3 cells, vimentin was downregulated (Fig. 4B and C). These results supported the notion that SET8 and ZEB1 are required for trans-repression of E-cadherin and trans-activation of vimentin. It appeared that SET8 functions in a dual mode in ZEB1-regulated gene expression.

SET8-induced H4K20mel has a dual function in ZEB1-regulated gene expression. qChIP assays using anti-H4K20me1, anti-H4K20me2 and anti-H4K20me3 in PC-3 
cells indicated that H4K20 was mono-, di- and trimethylated in E-cadherin and vimentin promoters (Fig. 5A). To explore whether the monomethylation of H420 was catalyzed by SET8 in the promoter of E-cadherin and vimentin, SET8 was silenced in PC-3 cells and the methylation status of H4K20 in E-cadherin and vimentin promoters was assessed by qChIP. The results indicated that knockdown of SET8 was associated with a sharp reduction of monomethylation of H4K20, while only a slight decrease in di- and trimethylation of H4K20 was observed in the E-cadherin (Fig. 5B) and vimentin promoters (Fig. 5C). Of note, ZEB1 knockdown also led to a marked decrease of H4K20me1 in the E-cadherin (Fig. 5B) and vimentin (Fig. 5C) promoters. These results further supported the hypothesis that SET8 is recruited to the E-cadherin and vimentin promoters by ZEB1 to monomethylate H4K20. Collectively, these experiments indicated that SET8 is recruited to the E-cadherin promoter by ZEB1 to repress its transcription and to the vimentin promoter to activate its transcription through its H4K20 monomethylation activity.

\section{Discussion}

Cancer-cell invasion and metastasis are multistep processes comprising alterations in cell adhesion as well as transformation of cells to phenotypes with enhanced invasive and migratory potential (23). E-cadherin generally functions as a tumor suppressor gene and maintains the polarity as well as structural integrity of epithelial cell. Loss or dysfunction of E-cadherin is closely associated with tumorigenesis, invasion and metastasis in numerous cancer types.

The present study demonstrated that depletion of SET8 inhibits PC-3-cell migration and invasion in vitro; furthermore, silencing of SET8 in non-transformed PZ-HPV-7 prostate epithelial cells was shown to disrupt their epithelial-type morphology. Silencing of SET8 in PC-3 cells led to decreases in the expression of the epithelial markers E-cadherin and $\alpha$-catenin, and increased the expression of the mesenchymal markers $\mathrm{N}$-cadherin and vimentin implying that SET8 promotes EMT and enhances the invasive capacity of PC-3 PCa cells. Furthermore, SET8 was shown to exert a dual transcriptional regulatory function comprising the repression of E-cadherin expression and the activation of vimentin expression. A co-IP assay indicated that SET8 mediates the EMT by physically binding to the EMT-inducing transcription factor ZEB1.

The important role of SET8 as a histone H4K20-specific methyltransferase was further proved by its binding toanti-H4K20mel in the promoter regions of E-cadherin and vimentin. Furthermore, ZEB1 knockdown also led to a marked decrease of H4K20me1 in the E-cadherin and vimentin promoters. Although SET8 directly targeted the promoters of E-cadherin and vimentin, binding with ZEB1 resulted in a more marked change on the these promoters, which indicated that ZEB1 may enhance the enzyme activity of SET8. The present study revealed a role for SET8 in facilitating EMT and the invasive potential of PCa cells, suggesting that SET8 represents a potential therapeutic target for treating or preventing metastasis of PCa.

In conclusion, the present study revealed that SET8 is a positive effector of the EMT and PCa metastasis, by directly repressing the transcription of E-cadherin and activating that of vimentin via methylating H4K20me1; furthermore, it physically binds to ZEB1. ZEB1 is a transcription factor, the function of which is to directly bind DNA, and since the physical association of ZEB1 with SET8 was determined in the present study, it was reasonable to hypothesize that ZEB1 cooperates with SET8 to enhance prostate cancer cell metastasis. SET8 represents a promising therapeutic target for reversing or preventing EMT and tumor metastasis, which are major events in PCa progression and aggravation.

However, it remains elusive whether SET8 has any additional molecular targets involved in PCa-cell proliferation and invasion. It is known that neoplastic progression is attributable to a large number of genes; therefore, it is necessary to further explore the potential role of SET8 in the genesis and progression of PCa.

\section{Acknowledgements}

This study was supported by grants from the Zhejiang Provincial Foundation of National Science (no. LY13H160030) and the Scientific and Technological Developing Scheme of Hangzhou (no. 20130633B33).

\section{References}

1. Beltran H, Beer TM, Carducci MA de Bono J, Gleave M, Hussain M, Kelly WK, Saad F, Sternberg C and Tagawa ST: New therapies for castration-resistant prostate cancer: Efficacy and safety. Eur Urol 60: 279-290, 2011.

2. Martin GS: Cell signaling and cancer. Cancer Cell 4: 167-174, 2003.

3. Kase S, Sugio K, Yamazaki K, Okamoto T, Yano T and Sugimachi K: Expression of E-cadherin and beta-catenin in human non-small cell lung cancer and the clinical significance. Clin Cancer Res 6: 4789-4796, 2000.

4. Hay ED: An overview of epithelio-mesenchymal transformation. Acta Anat (Basel) 154: 8-20, 1995.

5. Kalluri R and Weinberg RA: The basics of epithelial-mesenchymal transition. J Clin Invest 119: 1420-1428, 2009.

6. Cano A, Perez-Moreno MA, Rodrigo I, Locascio A, Blanco MJ, del Barrio MG, Portillo F and Nieto MA: The transcription factor snail controls epithelial-mesenchymal transitions by repressing E-cadherin expression. Nat Cell Biol 2: 76-83, 2000.

7. Hajra KM, Chen DY and Fearon ER: The SLUG zinc-finger protein represses E-cadherin in breast cancer. Cancer Res 62: 1613-1618, 2002.

8. Smit MA and Peeper DS: Zeb1 is required for TrkB-induced epithelial-mesenchymal transition, anoikis resistance and metastasis. Oncogene 30: 3735-3744, 2011.

9. Comijn J, Berx G, Vermassen P, Verschueren K, van Grunsven L, Bruyneel E, Mareel M, Huylebroeck D and van Roy F: The two-handed $\mathrm{E}$ box binding zinc finger protein SIP1 downregulates E-cadherin and induces invasion. Mol Cell 7: 1267-1278, 2001.

10. Yang J, Mani SA, Donaher JL, Ramaswamy S, Itzykson RA, Come C, Savagner P, Gitelman I, Richardson A and Weinberg RA: Twist, a master regulator of morphogenesis, plays an essential role in tumor metastasis. Cell 117: 927-939, 2004.

11. Singh S, Sadacharan S, Su S, Belldegrun A, Persad S and Singh G: Overexpression of vimentin: Role in the invasive phenotype in an androgen-independent model of prostate cancer. Cancer Res 63: 2306-2311, 2003.

12. Zhao Y, Yan Q, Long $X$, Chen $X$ and Wang Y: Vimentin affects the mobility and invasiveness of prostate cancer cells. Cell Biochem Funct 26: 571-577, 2008.

13. Kouzarides T: Chromatin modifications and their function. Cell 128: 693-705, 2007.

14. Shilatifard A: Chromatin modifications by methylation and ubiquitination: Implications in the regulation of gene expression. Ann Rev Biochem 75: 243-269, 2006.

15. Esteller M: Cancer epigenomics: DNA methylomes and histone-modification maps. Nat Rev Genet 8: 286-298, 2007. 
16. Gronbaek K, Treppendahl M, Asmar F and Guldberg P: Epigenetic Changes in cancer as potential targets for prophylaxis and maintenance therapy. Basic Clin Pharmacol Toxicol 103: 389-396, 2008.

17. Couture JF, Collazo E, Brunzelle JS and Trievel RC: Structural and functional analysis of SET8, a histone H4 Lys-20 methyltransferase. Genes Dev 19: 1455-1465, 2005.

18. Fang J, Feng Q, Ketel CS, Wang H, Cao R, Xia L, Erdjument-Bromage H, Tempst P, Simon JA and Zhang Y: Purification and functional characterization of SET8, a nucleosomal histone $\mathrm{H} 4$-lysine 20-specific methyltransferase. Curr Biol 12: 1086-1099, 2002.

19. Yang F, Sun L, Li Q, Han X, Lei L, Zhang H and Shang Y: SET8 promotes epithelial-mesenchymal transition and confers TWIST dual transcriptional activities. EMBO J 31: 110-123, 2012.
20. Li Z, Nie F, Wang S and Li L: Histone H4 Lys 20 monomethylation by histone methylase SET8 mediates Wnt target gene activation. Proc Natl Acad Sci USA 108: 3116-3123, 2011.

21. Talasz H, Lindner HH, Sarg B and Helliger W: Histone H4-lysine 20 monomethylation is increased in promoter and coding regions of active genes and correlates with hyperacetylation. J Biol Chem 280: 38814-38822, 2005.

22. Wong TS, Gao W and Chan JY: Transcription regulation of E-cadherin by zinc finger E-box binding homeobox proteins in solid tumors. Biomed Res Int 2014: 921564, 2014.

23. Park SI, Zhang J, Phillips KA, Araujo JC, Najjar AM, Volgin AY, Gelovani JG, Kim SJ, Wang Z and Gallick GE: Targeting SRC family kinases inhibits growth and lymph node metastases of prostate cancer in an orthotopic nude mouse model. Cancer Res 68: 3323-3333, 2008. 